\title{
Duitsland
}

\section{Deutsches Erbrecht}

\author{
Wolfgang Eule*
}

Die nachstehenden Ausführungen zur Stellung des überlebenden Ehepartners und zum Pflichtteilsrecht nach deutschem Erbrecht sind - auch für niederländische Notare - relevant, wenn der Erblasser nach dem 16.08.2015 mit gewöhnlichem Aufenthalt zum Zeitpunkt des Todes in Deutschland verstorben ist bzw. verstirbt (Art. 21 Europäische Erbrechtsverordnung (EuErbVO)) bzw. wenn ein deutscher Erblasser mit gewöhnlichem Aufenthalt zum Zeitpunkt des Todes zum Beispiel in den Niederlanden verstirbt, aber gemäß Artikel 22 EuErbVO die Anwendung des deutschen Erbrechts wirksam gewählt hat oder eine solche Wahl aufgrund einer Verfügung von Todes wegen vor dem 17.08.2015 gemäß Artikel 83 Absatz 4 EuErbVO fingiert wird.

Auch die vor dem 17.08.2015 erfolgten Wahlen des deutschen Erbrechts gemäß Artikel 5 Haager Erbrechtsübereinkommen durch Niederländer in Deutschland bzw. durch Deutsche in den Niederlanden werden bei Versterben nach dem 16.08.2015 gemäß Artikel 83 Absatz 2 EuErbVO anerkannt und es ist dann deutsches Erbrecht anzuwenden.

Das deutsche Erbrecht ist im Wesentlichen im 5. Buch des Bürgerlichen Gesetzbuches geregelt.

\section{Stellung des überlebenden Ehepartners}

Der zum Zeitpunkt des Todes des Erblassers mit diesem wirksam verheiratet gewesene Ehepartner ist immer gesetzlich erbberechtigt. Dies gilt seit dem 01.10.2017 auch für gleichgeschlechtliche Eheleute (Homo-Ehe).

Die nachstehenden Ausführungen gelten auch für den überlebenden Partner einer eingetragenen gleichgeschlechtlichen Lebenspartnerschaft und nach wohl herrschender Meinung auch für den überlebenden Partner einer verschiedengeschlechtlichen Lebenspartnerschaft (die es in Deutschland nicht gibt), weil Artikel 17 b Einführungsgesetz zum Bürgerlichen Gesetzbuche (EGBGB) darauf analog angewendet wird. Der Partner einer nichtehelichen, das heißt nicht formalisierten Lebensgemeinschaft, hat kein gesetzliches Erbrecht und somit auch kein Pflichtteilsrecht.

\subsection{Gesetzliche Erbfolge und Erbquoten}

Die Höhe der gesetzlichen Erbquote des Ehepartners ist davon abhängig, welche sonstigen Erben welcher Erbordnung vorhanden sind und von dem für die Ehe geltenden Güterstand. Letzteres ist eine Besonderheit des deutschen Ehegattenerbrechts.

Gemäß $§ 1931$ Absatz 1 Bürgerliches Gesetzbuch (BGB) erhält der überlebende Ehepartner immer 1/4 Erbanteil. Beim gesetzlichen Güterstand der Zugewinngemeinschaft erhöht sich diese gesetzliche Erbquote gemäß $§ 1371$ Absatz 1 BGB um ein weiteres 1/4 (sogenanntes Erhöhungsviertel) als pauschaler Zugewinnausgleich, falls sich der überlebende Ehegatte nicht für die Geltendmachung eines konkret berechneten Zugewinnausgleiches entscheidet.

Bei dem kaum noch vorkommenden Wahlgüterstand der Gütergemeinschaft verbleibt es bei dem Erbanteil von 1/4, wenn der Erblasser auch Kinder hinterlässt. Zum Nachlass gehört die Hälfte des gemeinschaftlichen Vermögens (Gesamtgut) sowie das dem Erblasser allein zustehende Vorbehalts- oder Sondergut. 
Bei dem Wahlgüterstand der Gütertrennung verbleibt es ebenfalls bei dem Erbanteil von 1/4, wenn mehr als zwei Abkömmlinge des Erblassers vorhanden sind. Er erhöht sich auf $1 / 2$, wenn ein Kind vorhanden ist, und auf $1 / 3$, wenn zwei Kinder vorhanden sind ( $\$ 1931$ Abs. 4 BGB).

Hinterlässt der Erblasser neben dem überlebenden Ehegatten auch Kinder (Erben der ersten Erbordnung, wozu auch nichteheliche und adoptierte Kinder gehören, nicht aber Pflegekinder), verbleibt es bei den oben angegebenen Erbquoten. Nach einem kinderlos verstorbenen Erblasser erbt der überlebende Ehepartner bei dem Güterstand der Zugewinngemeinschaft 3/4 Erbteil. Jeweils 1/8 Erbteil geht dann an die Eltern bzw. - falls diese vorverstorben sind - an die Geschwister.

Bei den Güterständen der Gütergemeinschaft bzw. Gütertrennung beträgt die Erbquote des überlebenden Ehepartners 1/2, die andere Hälfte geht dann an die Eltern bzw. Geschwister.

Hinterlässt ein kinderlos verstorbener Erblasser weder Eltern noch Geschwister noch Großeltern, erbt der überlebende Ehepartner gemäß § 1931 Absatz 2 BGB allein.

\subsection{Gewillkürte Erbfolge}

Wird der überlebende Ehegatte durch Verfügung von Todes wegen des Erblassers zum (Allein-)Erben bestimmt, können die Kinder des Erblassers bzw. dessen Eltern, wenn er kinderlos verstorben ist, ihre Pflichtteilsansprüche (siehe dazu gleich Teil 2) sofort geltend machen und Auszahlungen verlangen. Eine Schutzregelung wie in Artikel 4:82 Burgerlijk Wetboek (BW) gibt es in Deutschland nicht. Will in Deutschland ein Erblassers den überlebenden Ehepartner insoweit schützen, kann und muss er mit seinen volljährigen und geschäftsfähigen Kindern bzw. mit den Eltern einen Pflichtteilsverzichtsvertrag gemäß $§ 2346$ Absatz 2 BGB schließen (siehe dazu gleich Teil 3).

\subsection{Ausschluss vom gesetzlichen und gewillkürten Ehegattenerbrecht bei Tod während des Scheidungsverfahrens}

$\mathrm{Zu}$ beachten ist eine weitere Besonderheit des deutschen Ehepartnererbrechts. Das gesetzliche und auch das gewillkürte Erbrecht des überlebenden Ehepartners entfällt nicht erst mit rechtskräftiger Scheidung der Ehe, sondern gemäß $\$$ 1933, 2077 BGB bereits dann, wenn der Erblasser selbst einen Scheidungsantrag beim Familiengericht gestellt hat bzw. sich wirksam dem Scheidungsantrag des überlebenden Ehepartners angeschlossen hat und die Ehe geschieden worden wäre, wenn der Erblasser nicht während des Scheidungsverfahrens verstorben wäre.

\subsection{Passive Vererblichkeit des}

\section{Ehegattenunterhaltsanspruches}

Ein besonderes Recht steht dem geschiedenen bzw. getrenntlebenden Ehepartner gegen den/die Erben zu. Er kann gemäß $§ 1586$ b BGB den Unterhaltsanspruch, der ihm/ihr bei Tod des unterhaltsverpflichteten Erblassers zustand, weiterhin (eventuell neu berechnet) verlangen, allerdings quantitativ beschränkt auf die Höhe des fiktiven Pflichtteilsanspruches zum Zeitpunkt des Todes ohne Berücksichtigung des Güterrechts (sogenannte passive Vererblichkeit des Ehegattenunterhaltsanspruches).

\section{Pflichtteilsrechte und pflichtteilsähnliche Rechte}

\subsection{Ordentlicher Pflichtteilsanspruch}

Trotz der verfassungsrechtlich garantierten Testierfreiheit wird diese am schwerwiegendsten durch das für nächste Angehörige des Erblassers bestehende Pflichtteilsrecht eingeschränkt. Auch das grundsätzlich bedarfsunabhängige Pflichtteilsrecht der Abkömmlinge ist verfassungsrechtlich garantiert (Bundesverfassungsgericht vom 19.04.2005 = Neue Juristische Wochenschrift 2005, 1561). Ob dies auch für die weiteren Pflichtteilsberechtigten, das heißt die Eltern des Erblassers und den Ehe- bzw. Lebenspartner gilt, ist nicht höchstrichterlich entschieden, aber wohl anzunehmen.

Anders als im niederländischen Erbrecht sind also im deutschen Erbrecht neben den Abkömmlingen des Erblassers auch der überlebende Ehepartner bzw. Lebenspartner einer eingetragenen Lebenspartnerschaft und die Eltern des Erblassers, wenn dieser kinderlos verstirbt, pflichtteilsberechtigt.

Bei Vorversterben eines pflichtteilsberechtigten Kindes steht auch dessen Kindern, das heißt den Enkelkindern des Erblassers, das Pflichtteilsrecht zu. Das Pflichtteilsrecht eines Kindes besteht getrennt und gesondert nach jedem Elternteil.

Der Pflichtteilsanspruch entsteht gemäß $§ 2317$ BGB mit dem Tod des Erblassers und ist danach vererblich und übertragbar.

Die Pflichtteilsquote ist die Hälfte der gesetzlichen Erbquote und der Pflichtteilsanspruch ein Geldanspruch gegen den/die Erben, berechnet nach dem Wert des vorhandenen Nachlassvermögens des Erblassers abzüglich der Erblasserschulden und der Beerdigungskosten.

Wenn für die Ehe des Erblassers mit dem überlebenden (Ehe-)Partner der Güterstand der Zugewinngemeinschaft galt, hat der überlebende (Ehe-)Partner ein Wahlrecht zwischen dem 'kleinen Pflichtteil', das heißt 1/8 Pflichtteilsquote (= 1/2 von $1 / 4)$ zuzüglich eines konkret berechneten Zugewinnausgleichsanspruches oder den 'großen Pflichtteil', das heißt in Höhe der Hälfte des gemäß $§ 1371$ Absatz 1 BGB erhöhten Erbteils, neben Kindern also eine Pflichtteilsquote von $1 / 4$ (= $1 / 2$ von 1/2). Diese Wahl des überlebenden (Ehe-)Partners hat auch Auswirkungen auf die Pflichtteilsquoten der anderen Pflichtteilsberechtigten. 
Um den Pflichtteilsanspruch berechnen zu können, hat der Pflichtteilsberechtigte einen Auskunftsanspruch gemäß $\$ 2314$ BGB gegen den/die Erben. In letzter Zeit wird insoweit die Vorlage eines von einem Notar erstellten Nachlassverzeichnisses verlangt. Die von der Rechtsprechung aufgestellten Anforderungen an ein solches notarielles Nachlassverzeichnis sind sehr weitgehend und können von Notaren zuweilen nicht erfüllt werden.

Grundsätzlich verliert ein pflichtteilsberechtigter Erbe sein Pflichtteilsrecht, wenn er die Erbschaft ausschlägt. Eine Ausnahme besteht aber gemäß $§ 2306$ BGB dann, wenn sein Erbteil belastet wird, zum Beispiel durch eine Testamentsvollstreckung, durch eine Vor- und Nacherbschaft oder eine Teilungsanordnung.

Eine einseitige Pflichtteilsentziehung durch den Erblasser durch Verfügung von Todes wegen ist gemäß § 2333, 2336 BGB nur unter sehr engen Voraussetzungen möglich und zulässig (zum Beispiel bei schweren Vergehen gegen Leib und Leben des Erblassers bzw. von dessen Ehepartner oder anderen Kindern).

Der Pflichtteilsanspruch verjährt gemäß §§ 195, 199 BGB nach Ablauf von drei Jahren. Die Verjährungsfrist beginnt gemäß $§ 199$ Absatz 1 BGB ab dem Schluss des Jahres, in dem der Pflichtteilsberechtigte Kenntnis von seiner Enterbung bzw. von seinen erbrechtlichen Ansprüchen erhalten hat. Längstens beträgt die Verjährungsfrist gemäß $§ 199$ Absatz 3 a) BGB dreißig Jahre.

\subsection{Pflichtteilsergänzungsanspruch}

Neben bzw. zusätzlich zum ordentlichen Pflichtteilsanspruch haben Pflichtteilsberechtigte auch einen selbständigen Pflichtteilsergänzungsanspruch gegen den/die Erben bzw. eventuell auch gegen den beschenkten Dritten, wenn der Erblasser innerhalb von 10 Jahren vor seinem Tod Vermögen verschenkt hat und dies somit nicht mehr zum Nachlassvermögen gehört. Diese 10-Jahresfrist gilt nicht bei Schenkungen an den Ehe- bzw. Lebenspartner oder wenn sich der Schenker bei der Schenkung das wirtschaftliche Eigentum zum Beispiel durch Nießbrauchsrecht oder Wohnungsrecht vorbehalten hat.

Der Pflichtteilsergänzungsanspruch besteht in Höhe der Pflichtteilsquote zum Zeitpunkt des Todes, mindert sich aber bei Tod nach dem 01.01.2010 um 10\% pro vollem Jahr seit der Schenkung (sogenannte Abschmelzung).

$\mathrm{Ob}$ der Pflichtteilsberechtigte bereits bei der Ausführung der Schenkung pflichtteilsberechtigt war, ist durch Änderung der Rechtsprechung nicht mehr relevant.

Der/die Erben, die nichts geschenkt erhalten haben, werden somit durch den Pflichtteilsergänzungsanspruch doppelt belastet. Sie erhalten weniger Nachlassvermögen und müssen einen erhöhten Pflichtteilsanspruch an den/die Pflichtteilsberechtigten bezahlen, was den Anteil am Nachlassvermögen nochmals mindert.

\subsection{Pflichtteilsrestanspruch}

Hat ein Pflichtteilsberechtigter durch Verfügung von Todes wegen des Erblassers weniger zugeteilt erhalten als seinem Pflichtteilsanspruch entspricht, kann er gemäß $§ 2305$ BGB einen Pflichtteilsrestanspruch geltend machen, das heißt einen Geldanspruch, der der Differenz zwischen dem Zugewendetem und dem Pflichtteilsanspruch entspricht.

\subsection{Pflichtteilsähnliche Rechte}

a. Nur bei gesetzlicher Erbfolge stehen dem überlebenden Ehepartner gemäß $\S 1932$ BGB bzw. dem über-

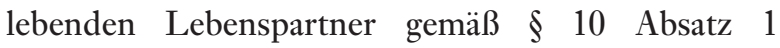
Lebenspartnerschaftsgesetz unabhängig vom Güterstand die zum ehelichen Haushalt gehörenden Gegenstände und die Hochzeitsgeschenke als Voraus zu, wenn sie mit dem Erblasser zum Zeitpunkt von dessen Tod einen gemeinschaftlichen Haushalt geführt haben. Neben Kindern des Erblassers besteht dieser Anspruch nur, wenn er zur Führung eines angemessenen Haushaltes weiterhin benötigt wird. Der überlebende (Ehe-)Partner kann diesen Anspruch wie ein Vermächtnis geltend machen.

b. Unabhängig vom Erbrecht kann der überlebende (Ehe-)Partner im Wege der Sonderrechtsnachfolge kraft Gesetzes die Fortsetzung des Mietverhältnisses an der zum Zeitpunkt des Todes gemeinsam mit dem Erblasser bewohnten Mietwohnung vom Vermieter verlangen ( $\$ 563 \mathrm{ff}$. BGB).

c. In den ersten 30 Tagen nach dem Tod des Erblassers können die zum Hausstand gehörenden Familienangehörigen im Wege des gesetzlichen Vermächtnisses gemäß § 1969 BGB verlangen, dass der Erbe bzw. die Erben ihnen den gleichen Unterhalt gewähren muss, den der Erblasser zum Zeitpunkt seines Todes schuldete (sogenannter Dreißigster). Ferner kann die Benutzung der Wohnung und der Haushaltsgegenstände für 30 Tage verlangt werden.

\section{Wirkung eines lebzeitigen Erb- und/oder Pflichtteilsverzichtsvertrages bei Wechsel des Erbstatuts zwischen Abschluss des Vertrages und Tod}

In Deutschland kommt es im Rahmen des 'estate planning' häufig vor, dass gesetzliche erb- und pflichtteilsberechtigte Verwandte auf diese Rechte gemäß § 2346 BGB verzichten, mit oder ohne Zahlung einer Abfindung.

Ein solcher Verzichtsvertrag zwischen dem zukünftigen Erblasser und dem/den Berechtigten bedarf gemäß $\S 2348$ BGB der notariellen Beurkundung, bei der der 
zukünftige Erblasser persönlich anwesend sein muss. Die Verzichtenden können sich vertreten lassen und den Vertrag anschließend genehmigen. Bei Verzicht durch Kinder gilt dieser auch für deren Abkömmlinge und beseitigt das Erb- bzw. Pflichtteilsrecht des Verzichtenden mit dinglicher Wirkung.

Ein Verzichtsvertrag ist seit dem 17.08.2015 gemäß Artikel 3 Absatz 1 b) EuErbVO ein einseitiger Erbvertrag gemäß Artikel 25 Absatz 1 EuErbVO bzw. ein mehrseitiger Erbvertrag gemäß Artikel 25 Absatz 2 EuErbVO. Es gilt das für den potentiellen Erblasser objektiv gemäß Artikel 21 EuErbVO bzw. das aufgrund einer Rechtswahl gemäß Artikel 22 EuErbVO anzuwendende Recht zum Zeitpunkt des Vertragsabschlusses (sogenanntes Errichtungsstatut). Auch Niederländer mit gewöhnlichem Aufenthalt in Deutschland können somit einen solchen Verzichtsvertrag schließen, es sei denn, sie haben gemäß Artikel 22 EuErbVO die Anwendung des niederländischen Erbrechts gewählt. Dann ist ein solcher Verzichtsvertrag unzulässig, weil er nach niederländischem Recht gemäß Artikel 4:4 Absatz 2 BW verboten ist.

Seit einigen Jahren wurde und wird in der Fachliteratur die Rechtsauffassung vertreten, dass ein solcher Verzichtsvertrag bei einem Wechsel des Erbstatuts beim Erblasser zwischen der Errichtung und dem Tod zwar zulässig und wirksam bleibt, aber keinerlei Wirkungen mehr hat, wenn zum Zeitpunkt des Todes für den Erblasser ein Erbrecht anzuwenden ist, das einen solchen Verzichtsvertrag verbietet, wie zum Beispiel das niederländisches Erbrecht gemäß Artikel 4:4 Absatz 2 BW.

Nach anderer - meines Erachtens zutreffender Rechtsauffassung - unterliegen auch die Wirkungen eines solchen Verzichtsvertrages dem Errichtungsstatut und nicht dem beim Tod des Erblassers geltenden Erbstatut. Verzichtende, die einen solchen Verzichtsvertrag freiwillig abgeschlossen haben, können sich somit nach einem Statutenwechsel zum Beispiel zum niederländischen Erbrecht (durch Umzug oder durch Rechtswahl gemäß Art. 22 EuErbVO seitens des Erblassers) nicht auf Artikel 4:4 Absatz 2 BW berufen.

Höhergerichtliche Rechtsprechung gibt es zu dieser Problematik noch nicht. Es ist daher zur Zeit unklar, ob ein wirksam und zulässig abgeschlossener Erb- und/ oder Pflichtteilsverzichtsvertrag noch Wirkungen hat, wenn ein niederländischer Erblasser, für den zum Zeitpunkt des Abschlusses dieses Verzichtsvertrages deutsches Erbrecht anzuwenden war, wieder in die Niederlande zurück verzieht.

Das gleiche Problem besteht natürlich auch bei deutschen Erblassern, die einen solchen Verzichtsvertrag zum Beispiel mit ihren Kindern geschlossen haben, wenn sie danach in die Niederlanden umziehen und mit gewöhnlichem Aufenthalt dort versterben. Deutsche Erblasser können diese Problematik aber umgehen, in dem sie die Anwendung des deutschen Erbrechts gemäß Artikel 22 EuErbVO wählen. 